\title{
Electroweak Precision Tests with a Future Linear Collider
}

\author{
Wolfgang Menges* Institut für Experimentalphysik, Universität Hamburg, Luruper \\ Chaussee 149, 22761 Hamburg, Germany \\ E-mail: Woiffgang. Menges@desy.dè
}

AbStRaCt: Future high energy linear electron positron colliders with centre-of-mass enegies between 90 and $1000 \mathrm{GeV}$ offer a unique opportunity to study precisely the Standard Model and phenomena from new physics. Most important, the mechanism of electroweak symmetry breaking can be established in full depth if one or more Higgs bosons are observed. Precision measurements of electroweak processes open a window into the multi$\mathrm{TeV}$ energy range for searching for new physics, which is of particular importance if no light Higgs boson is observed.

\section{Framework}

The physical motivation and the technical feasibility of linear electron-positron colliders (LC) has been intensively studied in the past years in Japan (JLC [i] and Europe (TESLA [ịi]). The energy of such a machine would be $500 \mathrm{GeV}$ in a first phase with upgrade potential to about $1 \mathrm{TeV}$.

A LC has a very rich physics potential covering both the study of new phenomena (Higgs physics, Supersymmetry and alternative physics) and precision tests of the Standard Model (SM) (top quark physics, electroweak physics and QCD). Here only prospects for precision Higgs measurements and precision electroweak measurements in the context of strong electroweak symmetry breaking (EWSB) will be described. The results presented here are based on integrated luminosities of 500 to $1000 \mathrm{fb}^{-1}$ and on realistic simulations of detector performance and background conditions.

\section{Higgs Physics}

In the SM and many of its extensions, EWSB proceeds via the Higgs mechanism leading to one or more physical Higgs bosons. The mass of the lightest Higgs boson is bounded

\footnotetext{
${ }^{*}$ Speaker.
} 
from above on theoretical grounds, requiring perturbativity of the Higgs coupling up to a high energy scale. Experimentally, $m_{\mathrm{H}}<200 \mathrm{GeV}$ is obtained from the interpretation of electroweak precision measurements within the SM.

While a Higgs boson, if it exists, is very likely to be found at Tevatron or LHC, the precise measurement of its properties and couplings can only be performed at the LC.

The main production mechanisms for the SM-Higgs boson at the LC are Higgsstrahlung $\left(\mathrm{e}^{+} \mathrm{e}^{-} \rightarrow \mathrm{ZH}\right)$ and WW-fusion $\left(\mathrm{e}^{+} \mathrm{e}^{-} \rightarrow \nu_{\mathrm{e}} \bar{\nu}_{\mathrm{e}} \mathrm{H}\right)$. This gives $\mathcal{O}\left(10^{5}\right)$ Higgs bosons per year for a light Higgs. Furthermore the Yukawa process $\mathrm{e}^{+} \mathrm{e}^{-} \rightarrow \mathrm{t} \overline{\mathrm{t}} \mathrm{H}$ and the double Higgsstrahlung process $\mathrm{e}^{+} \mathrm{e}^{-} \rightarrow$ ZHH can be exploited. The Higgs boson itself can be detected independent of its decay products through the observation of a mass peak in the invariant mass spectrum recoiling against a lepton pair from the Z-decay in the Higgsstrahlung process. Furthermore, all relevant Higgs boson decays $\left(\mathrm{b} \overline{\mathrm{b}}, \mathrm{c} \overline{\mathrm{c}}, \tau^{+} \tau^{-}, \mathrm{gg}, \mathrm{W}^{+} \mathrm{W}^{-}, \mathrm{ZZ}, \gamma \gamma, \mathrm{t} \overline{\mathrm{t}}\right)$ can be observed exclusively in the detector under study.

This large variety of accessible production mechanisms and decay modes in conjunction with the well defined initial state and clean environment of $\mathrm{e}^{+} \mathrm{e}^{-}$-collisions allows the determination of the complete profile of the observed Higgs particle.

The coupling gHzZ of the Higgs boson to the $\mathrm{Z}$ can be derived in a model independent way from the observed cross-section of the Higgsstrahlung process with leptonically decay-

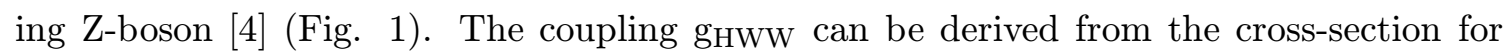

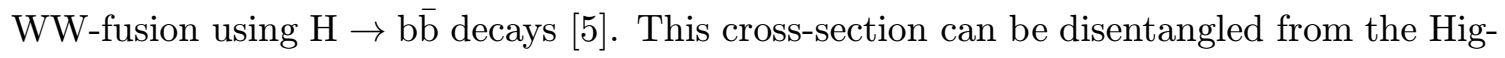
gsstrahlung contribution with $\mathrm{Z} \rightarrow \nu \bar{\nu}$ exploiting the different distributions of the missing invariant mass (Fig. $\overline{2}$,

An essential prediction of the Higgs mechanism is the Yukawa coupling of the Higgs

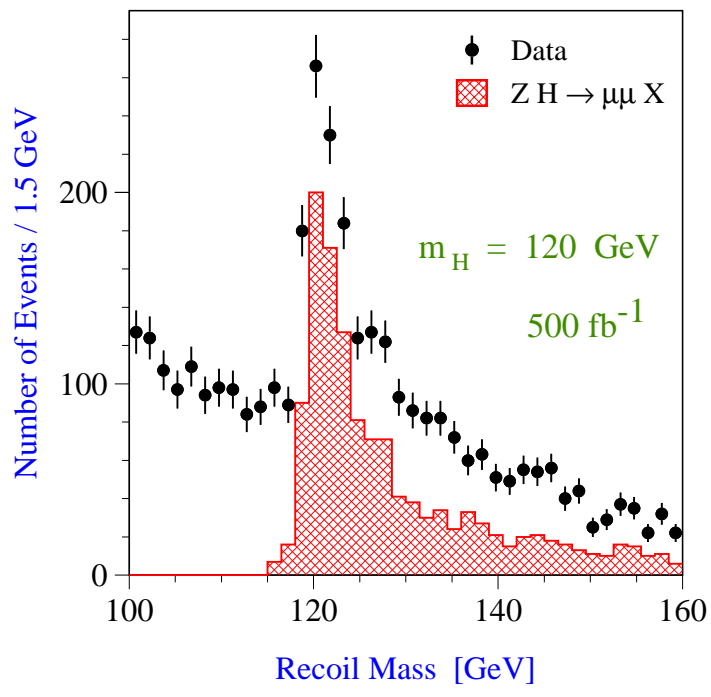

Figure 1: Higgs boson signal in the recoil mass distribution to a muon pair compatible with a $\mathrm{Z}$ decay for a simulated Higgs boson mass $m_{H}=120 \mathrm{GeV}$ and $500 \mathrm{fb}^{-1}$ at $\sqrt{s}=$ $350 \mathrm{GeV}$.

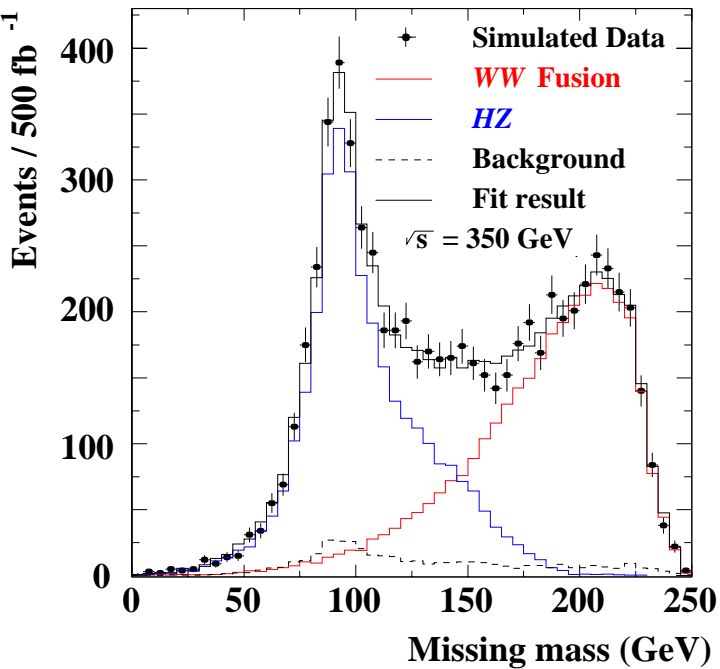

Figure 2: Missing mass distribution in events with two b-jets with an invariant mass compatible with the Higgs boson mass for $500 \mathrm{fb}^{-1}$ at $\sqrt{s}=350 \mathrm{GeV}$. 


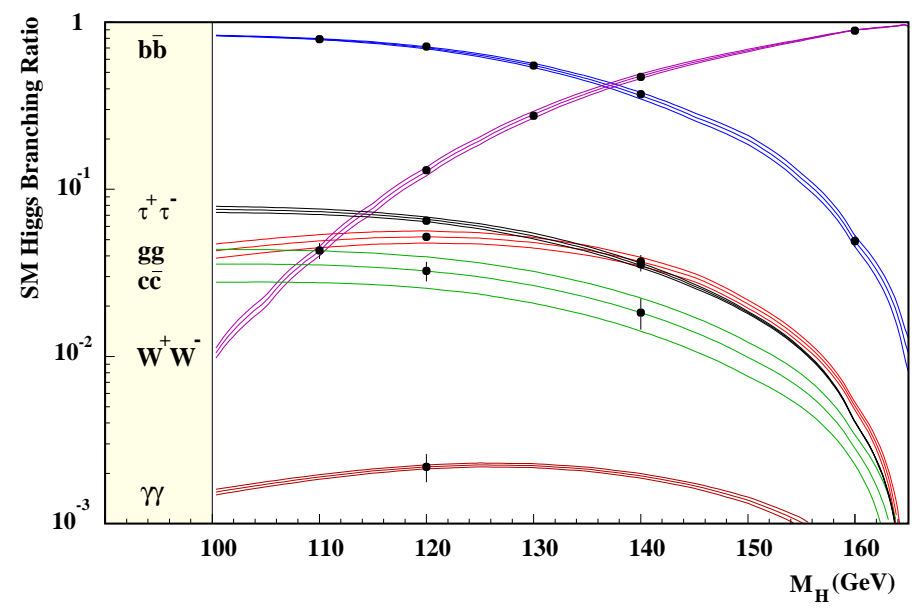

Figure 3: The SM prediction for the decay branching ratios of the Higgs boson. The points with error bars indicate the achievable experimental precision for $500 \mathrm{fb}^{-1}$. The bands indicate the theoretical uncertainties of the prediction.

boson to fermions being proportional to the fermion mass. This prediction can be accurately tested by measuring the Higgs boson decay branching ratios. The different hadronic Higgs boson decays b $\bar{b}, c \bar{c}$ and gg as well as $\tau^{+} \tau^{-}$can be disentangled using the excellent flavour tagging capabilities of a LC detector [6]. The expected precision of the branching ratio measurement is ranging from $2 \%$ (for $\mathrm{b} \overline{\mathrm{b}}$ ) to approximately $10 \%$ (for gg) for a light Higgs boson $\left(m_{\mathrm{H}}<160 \mathrm{GeV}\right)$ (Fig. '等).

The double Higgsstrahlung process $\mathrm{e}^{+} \mathrm{e}^{-} \rightarrow$ ZHH gives access to the triple Higgs coupling $\lambda_{\mathrm{HHH}}$ of the Higgs boson. This coupling determines the shape of the Higgs potential which is exactly predicted in terms of the Higgs mass in the SM and thus provides a rigorous test of the Higgs mechanism.

Since the cross-section for this process is small $\left(0.35 \mathrm{fb}\right.$ for $m_{\mathrm{H}}=120 \mathrm{GeV}$ at $\left.\sqrt{s}=500 \mathrm{GeV}\right)$, a very high luminosity is essential. A realistic study shows that $20 \%$ precision of $\lambda_{\mathrm{HHH}}$ seems possible for a light Higgs boson $\left[\overline{i_{1}}\right]$.

From the precision measurements of cross-sections and branching ratios the couplings of the Higgs boson to the different gauge bosons and fermions can be extracted [8i]. The expected precision ranges from $1.2 \%$ (for $g_{\mathrm{HzZ}}$ ) up to $3.7 \%$ (for $g_{\mathrm{Hc} \bar{c}}$ ) for a light Higgs boson $\left(m_{\mathrm{H}}=120 \mathrm{GeV}\right)$. As an example, Figure ${ }_{-1}^{1}$ shows the sensitivity of the couplings $g_{\mathrm{Hbb}}$ and $g_{\mathrm{Hcc}}$ at a LC to the mass of the pseudoscalar Higgs boson A in the MSSM.

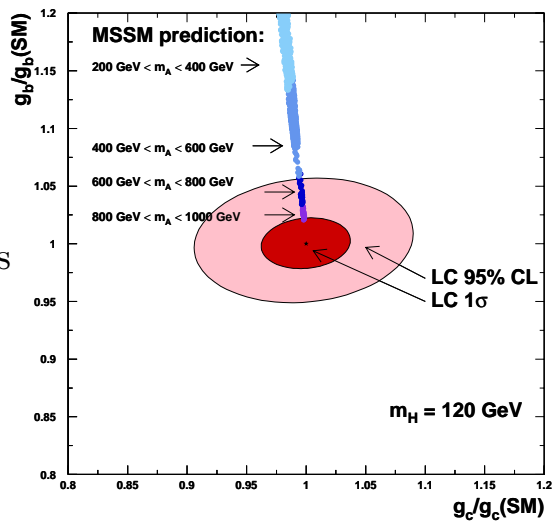

Figure 4: Expected sensitivity of the Higgs couplings $g_{\mathrm{Hbb}}$ vs. $g_{\mathrm{Hbb}}$ for a Higgs bosons mass $m_{\mathrm{H}}=120 \mathrm{GeV}$ and $500 \mathrm{fb}^{-1}$. 


\section{Strong Electroweak Symmetry Breaking}

Even if no light Higgs boson is found, the LC can contribute to the understanding of EWSB and the search for new physics. Unitarity requires that the interaction of gauge bosons becomes strong at high energies. Without knowing the details of the new strong interaction, an effective Lagrangian can be used to describe the interactions of electroweak gauge bosons [igi] . Its coupling parameters $\alpha_{1}, \alpha_{2}$ and $\alpha_{3}$ describe non-standard triple gauge boson couplings (TGC), and $\alpha_{4}$ and $\alpha_{5}$ describe non-standard quartic gauge boson couplings (QGC), assuming $\mathrm{SU}(2)_{c}$ invariant and linearly breaking operators, which conserve $\mathrm{C}$ and P. Each $\alpha_{i}$ is related to an energy scale of new physics $\Lambda_{i}^{*}$ by

$$
\frac{\alpha_{i}}{16 \pi^{2}}=\left(\frac{246 \mathrm{GeV}}{\Lambda_{i}^{*}}\right)^{2}
$$

The couplings are normalised in such a way that they are of $\mathcal{O}(1)$ for new physics around $3 \mathrm{TeV}$.

TGCs can be measured in the most general way in $\mathrm{W}$-pair production. A realistic study of the semi-leptonic decay channel $\mathrm{W}^{+} \mathrm{W}^{-} \rightarrow \mathrm{q} \overline{\mathrm{q}} l \bar{\nu}_{l}$ at an energy of $800 \mathrm{GeV}$ shows that the expected errors for anomalous TGCs [1] $\left.\overline{1}_{1}^{1}\right] \Delta g_{Z}^{1}, \Delta \kappa_{\gamma}$ and $\lambda_{\gamma}$ are in the order of $10^{-4}[1 \overline{1} \overline{0}]$, which is up to two orders of magnitude better than at LEP [1]2i] or Tevatron. This includes the use of polarised electrons $( \pm 80 \%)$ and positrons $(\mp 60 \%)$.

The $\alpha$ parameters are a linear combination of $\Delta g_{Z}^{1}, \Delta \kappa_{Z}$ and $\Delta \kappa_{\gamma}$. Unfortunately this transformation is singular and only two out of three couplings can be measured independently in this process, resulting in a blind direction in the parameter space. Figure , TGC measurement. The expected errors for $\alpha_{2}$ and $\alpha_{3}$ can be interpreted by equation ( scale of new physics. Using electron polarisation $( \pm 80 \%)$ yields $8.7 \mathrm{TeV}$ for $\Lambda_{2}^{*}$ and $11.6 \mathrm{TeV}$ for $\Lambda_{3}^{*}$ at $800 \mathrm{GeV}$ at $68 \%$ confidence level assuming $\Delta \alpha_{1}=$ 0 . These limits can be increased by a factor of 1.2 using also positron polarisation $(\mp 60 \%)$.

The parameter $\alpha_{1}$ can be indepen-

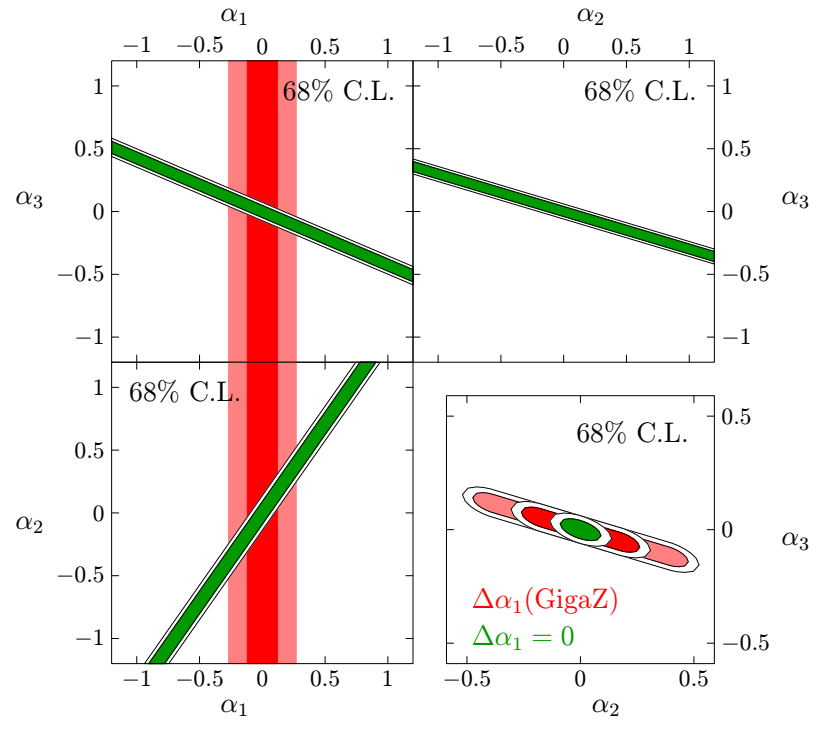

Figure 5: Sensitivity for the strong EWSB parameters $\alpha_{1,2,3}$ from a TGC measurement at $800 \mathrm{GeV}$ and $1000 \mathrm{fb}^{-1}$ using polarised electrons $( \pm 80 \%)$ and positrons $(\mp 60 \%)$.

dently obtained from electroweak precision observables at the Z-Pole. Figure the expected error on $\alpha_{1}$ from a measurement at GigaZ. This option of a LC describes a high luminosity run at the Z-pole $(\sqrt{s} \approx 91 \mathrm{GeV})$. A limit of $5.9 \mathrm{TeV}$ for $\Lambda_{1}^{*}$ at $68 \%$ confidence level can be obtained. 
The QGC parameters $\alpha_{4}$ and $\alpha_{5}$ can be measured in the processes $\mathrm{e}^{+} \mathrm{e}^{-} \rightarrow \nu_{\mathrm{e}} \bar{\nu}_{\mathrm{e}} \mathrm{W}^{+} \mathrm{W}^{-}$ and $\mathrm{e}^{+} \mathrm{e}^{-} \rightarrow \nu_{\mathrm{e}} \bar{\nu}_{\mathrm{e}}$ ZZ. A realistic study of the fully hadronic decay channel was performed at a centre-of-mass energy of $800 \mathrm{GeV}$ [1] ${ }_{1}^{1}$ in. The couplings are extracted by fitting the total cross-section and differential distributions. The use of left-handed electrons $(-80 \%)$ and right-handed positrons (40\%) improves the result significantly. The combination of both channels removes ambiguities and increases the sensitivity. The expected errors on $\alpha_{4}$ and $\alpha_{5}$ can again be interpreted as a limit on the energy scale of new physics. Using equation (1יב) gives $2.3 \mathrm{TeV}$ for $\Lambda_{4}^{*}$ and $3.1 \mathrm{TeV}$ for $\Lambda_{5}^{*}$ at $68 \%$ confidence level. The limits improve asymptotically as $1 / \sqrt{\mathcal{L}}$, where $\mathcal{L}$ is the integrated luminosity.

\section{Conclusion}

An electron positron linear collider in the $90-1000 \mathrm{GeV}$ regime allows very significant insights into physics at the electroweak scale. The essential parts of the Higgs mechanism of electroweak symmetry breaking can be fully established through precision measurements of Higgs boson properties. If no light Higgs boson is found, electroweak precision measurements of triple and quartic gauge boson couplings can extend the energy range for searches for new physics far into the multi-TeV energy range.

\section{References}

[1] K. Abe et al. [ACFA Linear Collider Working Group], Particle Physics Experiments at JLC, [hep-ph/0109166].

[2] T. Abe et al. [American Linear Collider Working Group], Linear Collider Physics Resource Book for Snowmass 2001, SLAC-R-570.

[3] J. A. Aguilar-Saavedra et al. [ECFA/DESY LC Physics Working Group], TESLA Technical Design Report Part III: Physics at an $e^{+} e^{-}$Linear collider, thep-ph/0106315.

[4] P. Garcia-Abia and W. Lohmann, 'Eur. Phys. J. Direct. C ${ }^{2} \mathbf{2}(2000)$ 1', hep-ex/9908065].

[5] K. Desch and N. Meyer, Study of Higgs Boson Production through WW Fusion at TESLA, LC-P

[6] M. Battaglia, Measuring Higgs Branching Ratios and Telling the SM from a MSSM Higgs Boson at the $e^{+} e^{-}$Linear Collider, [hep-ph/9910271].

[7] C. Castanier, P. Gay, P. Lutz and J. Orloff. Higgs Self Coupling Measurement in $e^{+} e^{-}$ Collisions at Center-of-Mass Energy of $500 \mathrm{GeV}$, LC-P HSM-2000-061' [hep-ex/0101028].

[8] K. Desch and M. Battaglia, Determination of the Higgs Profile: HFITTER,

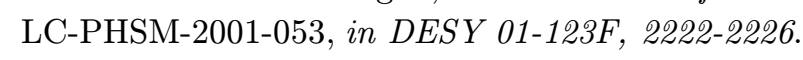

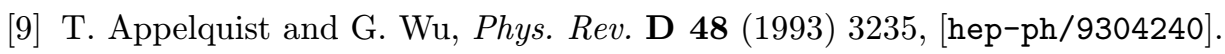

[10] W. Menges, A study of Charged Triple Gauge Couplings at TESLA, LC-P DESY 01-123F, 1635-1669.

[11] K. Hagiwara, R. D. Peccei, D. Zeppenfeld and K. Hikasa, Nucl. Phys. B 282

[12] S. Villa, these proceedings.

[13] R. Chierici, S. Rosati and M. Kobel, Strong Electroweak Symmetry Breaking Signals in WW Scattering at TESLA, LC-PHSM-2001-038', in DESY 01-123F, 1906-1923. 